\title{
PANDORA'S BOX
}

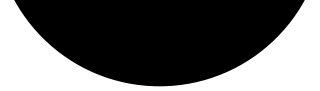

Pandora searches the world literature for evidence, news and other sources on matters of interest (doesn't shy away from controversy) to bring to the reader. She welcomes comments and suggestions (via ip@rcpsych. ac.uk)

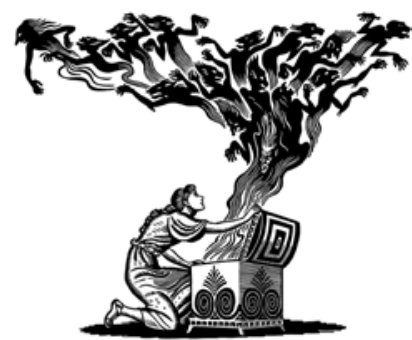

Food for thought - the Mediterranean diet (MeDi) is good for your brain!

1 he Mediterranean diet, high in vegetables and fish, is recognised not only for its beneficial effects on our general physical health but also for reducing the risk for cognitive impairment. But does it have any measurable effects on brain structure? In a cross-sectional study of older people (mean age 79.8 years), male and female, with normal cognitive function, those on a diet high in vegetables and fish had thicker cortices in the frontal, parietal and occipital lobes. However, a word of caution for those with a 'sweet tooth'. The study also found a decrease in the thickness of the entorhinal cortex in those who indulged in higher carbohydrate intake.

It would be interesting to see if prospective studies confirm all these findings but in the meantime Pandora will stick to MeDi and go easy on sugars!

Staubo, S. C., Aakre, J. A., Vemuri, P., et al (2016) Mediterranean diet, micronutrients and macronutrients, and MRI measures of cortical thickness. Alzheimer's and Dementia. doi: 10.1016/j. jalz.2016.06.2359. [Epub ahead of print]

\section{Turning off expression of the Huntington disease gene}

T he team running the FINGERS4CURE project, which is funded by the European Union and led by researchers at Imperial College London, have engineered a 'zinc finger', a protein targeting the huntingtin (HTT) gene, found in people with Huntington's disease, and repressing its ability to create toxic levels of harmful proteins in the brain.

Although the precise cause of the disease is still not known, it is hoped that turning off that gene's expression could be beneficial. The results from a trial in mice are encouraging. A single injection of the therapeutic protein reduced the activity of the gene for several months. Could there finally be a prophylactic treatment for this devastating condition?

Agustín-Pavón, C. Mielcarek, M., Garriga-Canut, M., et al (2016) Deimmunization for gene therapy: host matching of synthetic zinc finger constructs enables long-term mutant Huntingtin repression in mice. Molecular Neurodegeneration. doi:10.1186/s13024-016. 0128-x

\section{Keep your toddler's brain active!}

We can't retrieve memories of events that took place during our first 2-4 years of life, but do they matter? According to scientists, these infantile memories, which we cannot remember (infantile/ childhood amnesia), are important to our brain development. Experiments in rats show that early life experiences, although not remembered, can influence adult life behaviour.

This is a critical period for episodic learning, when the hippocampus becomes able to efficiently process and store memories in the long term, and which concerns important functions such as vision and language acquisition.

In a series of experiments it was found that if the hippocampus is inactive during this developmental stage, when the nervous system is especially sensitive to environmental stimuli, such latent memories formed could not be recalled in later life by reminders.

Essentially, during this critical period of early life, the brain learns how to form long-term memories. The brain needs stimulation to enable it to achieve this; if it fails to do so, the developmental ability of the neurological system is impaired. The authors suggest that using learning and environmental interventions during this critical period may help significantly in addressing intellectual disabilities.

Travaglia, A., Bisaz, R., Sweet, E. S., et al (2016) Infantile amnesia reflects a developmental critical period for hippocampal learning. Nature Neuroscience. doi: 10.1038/nn.4348. [Epub ahead of print] PMID:27428652

\section{Antisocial behaviour - brain maldevelopment or teenage rebellion?}

Teuroimaging that investigates structural covariance between brain regions is an increasingly used approach to investigating the synchronised maturation of different brain regions and the study of psychiatric disorders with presumed neurodevelopmental origins.

Researchers from the universities of Cambridge, Southampton and Rome used magnetic resonance imaging (MRI) to examine brain structure in males with a diagnosis of conduct disorder and healthy controls, aged 16-21 years. They calculated interregional correlations in cortical thickness as a measure of structural covariance in male youths with childhood-onset conduct disorder (CO-CD), adolescence-onset CD (AO-CD) and healthy controls, in two independent patient samples.

The CO-CD youths displayed more significant interregional correlations than the $\mathrm{AO}-\mathrm{CD}$ youths and the healthy controls, whereas the AO-CD individuals displayed fewer correlations than the healthy controls. The three groups differed in the strength of the interregional correlations across frontal, temporal, parietal and occipital regions.

The researchers' interpretation of the results is that they reflect specific disruptions in the development of the brain during adolescence. They concluded that this is compelling evidence that conduct disorder is a 'real psychiatric disorder' and not an 'exaggerated form of teenage rebellion'. Further research is needed to examine the factors leading to this abnormal pattern of brain development, such as exposure to early adversity, and investigate further how to use these results to help young people with conduct disorder.

Fairchild, G., Toschi, N., Sully, K., et al (2016) Mapping the structural organization of the brain in conduct disorder: replication of findings in two independent samples. Journal of Child Psychology and Psychiatry. doi: 10.1111/jcpp.12581

\section{Misuse of prescription drugs in the European Union}

T is known that prescription drugs are passed 1 around families and friends and in some cases there is also a commercial element! Until recently 